\title{
Dissociation of Genetic and Hormonal Influences on Sex Differences in Alcoholism-Related Behaviors
}

\author{
Jacqueline M. Barker, ${ }^{1}$ Mary M. Torregrossa, ${ }^{1}$ Arthur P. Arnold, ${ }^{2}$ and Jane R. Taylor ${ }^{1}$ \\ ${ }^{1}$ Department of Psychiatry, Yale University, Connecticut Mental Health Center, New Haven, Connecticut 06519, and ${ }^{2}$ Department of Integrative Biology and \\ Physiology, University of California, Los Angeles, Los Angeles, California 90095
}

\begin{abstract}
Differences between men and women in alcohol abuse prevalence have long been attributed to social and hormonal factors. It is, however, becoming apparent that sex differences in substance dependence are also influenced by genetic factors. Using a four core genotype mouse model that enables dissociation of chromosomal and gonadal sex, we show that habitual responding for alcohol reinforcement is mediated by sex chromosome complement independent of gonadal phenotype. After moderate instrumental training, chromosomal male (XY) mice became insensitive to outcome devaluation, indicating habitual responding. Chromosomal female (XX) mice remained sensitive to outcome devaluation, signifying goal-directed behavior. There was no effect of gonadal phenotype on habitual responding. Conversely, alcohol drinking was predicted by gonadal phenotype independent of sex chromosome complement. These results indicate that different alcoholism-related behaviors are determined independently by gonadal and chromosomal sex.
\end{abstract}

\section{Introduction}

Epidemiological studies report higher rates of alcohol dependence and abuse in men compared with women. Being male is consistently identified as a risk factor for alcohol abuse (Kalaydjian et al., 2009), though in rodent models, females have been shown to consume more alcohol than males in free-drinking paradigms (Lancaster et al., 1996; Middaugh et al., 1999). Men also develop more alcohol-related problems regardless of age or socioeconomic status, suggesting that biological factors are responsible for this disparity (Lynch et al., 2002). Addiction to alcohol involves a transition from casual use, where alcohol is sought for its rewarding properties, to habitual use where drinking is elicited by environmental stimuli (Jentsch and Taylor, 1999; Everitt and Robbins, 2005). This represents a shift from goal-directed (i.e., action-outcome) to habitual (i.e., stimulusresponse) behavior, and a shift from ventral corticostriatal circuitry involving prefrontal cortex and ventromedial striatum, to a more dorsal circuit involving dorsolateral striatum (Belin and Everitt, 2008). The transition from goal-directed to habitual responding has been shown to develop more rapidly for an alcohol reinforcer than for a natural reinforcer (Dickinson et al., 2002). Although the molecular mechanisms underlying the development of habitual responding are unclear, it is likely that changes in transmission at dopaminergic and glutamatergic synapses alter long-term synaptic plasticity, ultimately producing a shift in response strategy (Wang et al., 2007). Such response strategies can be differentiated based on their sensitivity to changes in reinforcer value. If an animal responds less for a reinforcer that has

Received Jan. 23, 2010; revised May 6, 2010; accepted May 26, 2010.

This research was supported by National Institutes of Health Grants AA017776, AA017537, AA012870, and DA022812.

Correspondence should be addressed to Jane R. Taylor at the above address. E-mail: jane.taylor@yale.edu.

DOI:10.1523/JNEUROSCI.0548-10.2010

Copyright $\odot 2010$ the authors $\quad 0270-6474 / 10 / 309140-05 \$ 15.00 / 0$ been "devalued," it is indicative of goal-directed behavior, while maintenance of responding indicates habit (Dickinson, 1985).

Understanding sex differences in maladaptive behaviors, such as inappropriate habitual responding, is critical for prevention and treatment of alcohol-related disorders. Traditionally, the organizational (permanent) and activational (nonpermanent) influence of gonadal hormones has been thought to underlie sex differences in behavior (Arnold and Gorski, 1984), but development of the four core genotype (FCG) mouse model has enabled exploration of the distinct influence of sex chromosome complement and gonadal phenotype (De Vries et al., 2002; Arnold and Chen, 2009). By deleting the portion of the Y chromosome containing the testes determining factor (Sry), which is responsible for initiation of testes development and therefore ultimately the production of testosterone, and inserting an Sry transgene onto an autosome, XYM (gonadal male) mice are produced. These mice father four different progeny in which gonadal sex (testes vs ovaries) and sex chromosome complement (XX vs XY) are independent: XX gonadal females (XXF), XX gonadal males (XXM), XY gonadal females (XYF), and XY gonadal males (XYM) (Arnold and Gorski, 2009). To detect activational effects of gonadal hormones on behavior, mice were either gonadectomized or sham-operated at $45 \mathrm{~d}$ of age. Although it is becoming clear that sex chromosome complement plays a critical role in determining neuronal activity and connectivity, the functional outcomes of such differences are not yet well understood. Here, we used an FCG mouse model to investigate the independent roles of sex chromosome complement, gonadal phenotype, and hormonal status on alcohol drinking and habit formation.

\section{Materials and Methods}

Subjects. Mice were MF1 FCG mice in which the testis-determining gene, Sry, is deleted from the Y chromosome. In breeders, an Sry transgene is inserted onto an autosome, resulting in testis formation. Because the Sry transgene is on an autosome, it segregates independently of sex chromo- 
some (De Vries et al., 2002; Arnold and Chen, 2009). XYM (gonadal and chromosomal male) mice are then bred with intact XX (gonadal and chromosomal female) mice to produce the four core genotypes: The two parent genotypes as well as XYF (chromosomal male, gonadal female) and XXM (chromosomal female, gonadal male). By comparing these genotypes, we can segregate the role of chromosome complement and gonadal hormone influences.

Mice were genotyped and underwent gonadectomy (GDX) or sham GDX at $45 \mathrm{~d}$ of age. Surgeries were conducted at the University of California, Los Angeles and were approved by the University of California, Los Angeles Institutional Animal Care and Use Committee (IACUC). Between 60 and $90 \mathrm{~d}$ of age, mice were shipped to Yale University where all behavioral analyses were conducted. All behavioral procedures were approved by the Yale University IACUC.

Instrumental acquisition. Mice were food restricted to $90 \%$ of freefeeding weight for the duration of the experiment. Water was available ad libitum. Before instrumental training, mice received $30 \mathrm{~min}$ exposure to $10 \%$ alcohol in a novel cage. Intake was measured to assure that all animals consumed alcohol and to prevent neophobic reactions during training.

Instrumental chambers were standard chambers, identical to those described by Quinn et al. (2007). Briefly, 12 mouse instrumental chambers ( $15 \mathrm{~cm}$ deep $\times 17 \mathrm{~cm}$ wide $\times 12 \mathrm{~cm}$ high) housed within a soundattenuating box were used for these experiments (Med Associates). The side-walls of each chamber were made of stainless steel panels and the front door, ceiling and back wall were made of clear Plexiglas. Each chamber was equipped with a $28 \mathrm{~V}$ house light located at the top of the left side wall, three adjacent nosepoke apertures located at the bottom of the left side wall, and a magazine located at the bottom of the middle panel on the right side wall. Liquid reinforcers were presented in the magazine via a motorized dipper the held $50 \mu \mathrm{l}$ of liquid. Nosepoke apertures and magazine were equipped with a light and photobeam sensor. A fan provided background noise and ventilation.

The training schedule used is based on that used by Quinn et al. (2007) to achieve "moderate training" that only produces habitual responding in sensitive populations. Briefly, training consisted of $1 \mathrm{~d}$ of magazine training, $3 \mathrm{~d}$ fixed ratio 1 (FR1) training, $3 \mathrm{~d}$ random interval $30 \mathrm{~s}$ (RI30) training and $3 \mathrm{~d}$ random interval $60 \mathrm{~s}$ (RI60) training. Magazine training was $30 \mathrm{~min}$ in length. All other training sessions were $45 \mathrm{~min}$ in duration.

Magazine training. Magazine training was performed to habituate mice to the operant chambers and to associate alcohol delivery with the magazine. Every 60 s, 10\% alcohol was presented via dipper. The dipper was available for $15 \mathrm{~s}$, during which the mouse had access to $\sim 50 \mu \mathrm{l}$ of alcohol. After $15 \mathrm{~s}$ the dipper retracted. Alcohol consumption was not measured during this task. However, mice were exposed to $10 \%$ ethanol in a pretraining exposure session and were found to readily consume the unsweetened ethanol solution as has been reported for other strains of mice (Zou et al., 2009).

Fixed ratio training. Either the left or right nosepoke aperture was designated as the "active" nosepoke, where responses could earn reinforcement. The other two nosepokes were designated the "inactive" nosepokes. On the FR1 schedule, an alcohol reinforcer was available for $5 \mathrm{~s}$ after each active nosepoke for the entire $45 \mathrm{~min}$ session.

Random interval training. RI training was identical to FR training except that the availability of reinforcement was changed. During RI training, reinforcement became available on a random 30 or $60 \mathrm{~s}$ schedule. Reinforcement could be earned every 30 or 60 s on average; however, the computer program randomly determined the actual duration of each interval. Therefore, reinforcement availability was not predictable. The first active nosepoke made after the interval elapsed resulted in an alcohol reinforcer. The duration of the next random interval was then generated automatically. This continued for the entire 45 min session.

Conditioned taste aversion. One day after the last RI60 instrumental acquisition session, mice were placed into a novel cage with ad libitum access to $10 \%$ alcohol in a water bottle and left undisturbed to consume alcohol. Immediately after removal from the cage, mice were injected with either $0.9 \%$ saline ("valued" mice) or $0.15 \mathrm{~m}$ lithium chloride (devalued mice; $40 \mathrm{ml} / \mathrm{kg}$, i.p.) and were then returned to their home cage. Approximately $3.5 \mathrm{~h}$ after injection, mice were allowed $1.5 \mathrm{~h}$ of home cage food access. Approximately $3 \mathrm{~h}$ following home cage feeding, valued mice received an injection of $0.15 \mathrm{~m}$ lithium chloride $(40 \mathrm{ml} / \mathrm{kg}$, i.p.) and devalued mice received an equal volume injection of $0.9 \%$ saline to normalize exposure to $\mathrm{LiCl}$ without pairing illness with alcohol or food chow in valued mice. This procedure was performed once daily across three consecutive days. Consumption was measured by weighing the bottles immediately before and after the $30 \mathrm{~min}$ session.

Habit testing. One day after the last conditioned taste aversion (CTA) session, testing was conducted in the same conditioning chamber used for instrumental acquisition. The test session lasted $5 \mathrm{~min}$ and was performed in extinction (no reinforcers were available), but was otherwise identical to conditions during acquisition. Performance in the $5 \mathrm{~min}$ test is depicted as a percentage of baseline responding with the rate of active nosepoking during the final training day serving as a baseline. Immediately following the instrumental test, animals received a final $30 \mathrm{~min}$ "posttest" consumption test to assess group differences in ad libitum alcohol consumption to verify that the CTA procedure was effective.

Locomotor behavior. One week after the habit testing session, locomotor behavior was assessed using the Accuscan Digiscan Micromonitor system, equipped with 10 photocells. Locomotor activity was measured by consecutive beam breaks. For baseline locomotor measures, mice received an injection of saline and were placed in clear plastic cages $(28 \times$ $17 \times 12 \mathrm{~cm}$ ) for $60 \mathrm{~min}$, and the amount of activity was collected in $5 \mathrm{~min}$ bins, on each of 3 consecutive days. The mean of these $3 \mathrm{~d}$ was stable and considered baseline locomotor activity. To measure locomotor response to alcohol, animals received an injection of $1.5 \mathrm{~g} / \mathrm{kg}$ alcohol immediately before being placed into a clean plastic cage. Locomotor activity was measured for $60 \mathrm{~min}$ on each day for $7 \mathrm{~d}$. Acute effects are reported on the first day of alcohol injection. After $7 \mathrm{~d}$ of alcohol injections, mice received no access to alcohol for 1 week. After 1 week, mice received a challenge injection of $1.5 \mathrm{~g} / \mathrm{kg}$ alcohol and locomotor activity was measured for $60 \mathrm{~min}$.

Statistical analyses. Analysis of data was performed using mixed-model ANOVA, with day of training or day of testing as the repeated measure for acquisition and CTA data, respectively. Data from the habit test and posttest consumption were analyzed using four-way ANOVA. Significant interactions and main effects were followed by Tukey's HSD. Results were considered significant if the probability of error was $<5 \%$.

\section{Results}

\section{Acquisition}

A mixed-model ANOVA revealed no main effects or interactions of sex chromosome complement, gonadal sex, GDX or devaluation on the number of reinforcers earned ( $p$ values $>0.1$ ). As expected, there was a main effect of day $\left(F_{(8,472)}=28.92, p<\right.$ $0.001)$, indicating that the number of reinforcers earned changed across training (Fig. 1a). A four-way ANOVA indicated no difference in the mean grams of alcohol earned relative to body weight across training sessions (Fig. $1 b$, inset; $p$ values $>0.15$ ). Although a mixed-model ANOVA suggested a gonadal sex by day of training interaction $\left(F_{(8,305)}=4.314, p<0.05\right)$, the post hoc analysis indicated an inconsistent pattern where there were only significant differences on day 2 of training $\left(F_{(1,37)}=3.18, p<\right.$ $0.05)$. Importantly, throughout training, all groups of mice received equivalent exposure to alcohol (mean grams per kilogram) as confirmed by a nonsignificant difference between the groups (Fig. $1 b$, inset).

A mixed-model ANOVA revealed main effects of sex chromosome complement and gonadal sex on the number of active nosepokes during acquisition $\left(F_{(1,489)}=31.60, p<0.001, F_{(1,489)}=\right.$ $16.47, p<0.001)$. There were no effects of GDX or devaluation on this measure ( $p$ values $>0.1$ ). Furthermore, there was a main effect of day $\left(F_{(8,481)}=4.84, p<0.001\right)$ indicating that the number of active nosepokes increased across training (Fig. 1c). Finally, a mixed-model ANOVA revealed no main effects or interactions of sex chromosome complement, gonadal sex, GDX or devalua- 
a

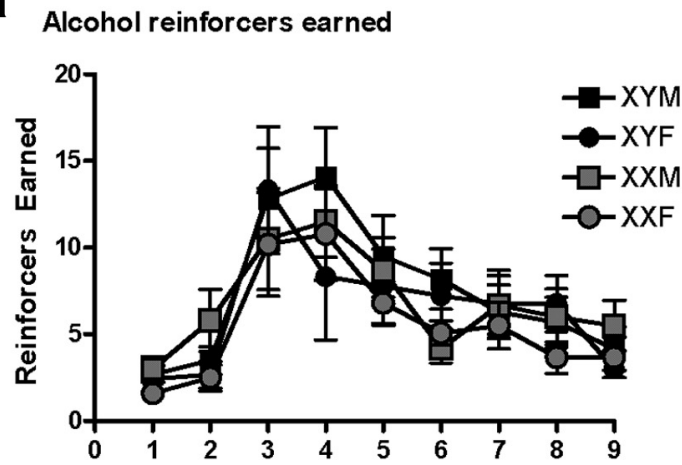

b

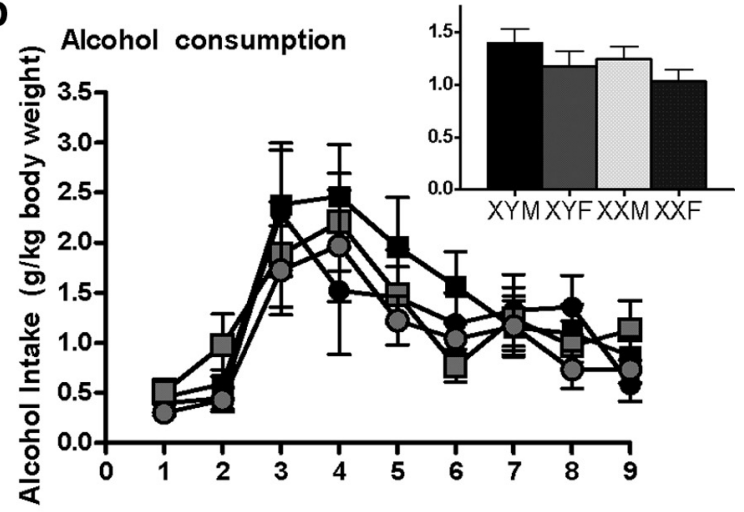

C Active response rate

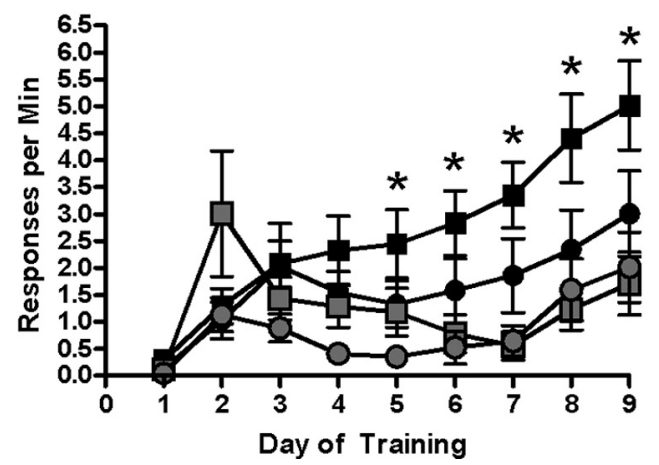

Figure 1. Mice did not differ in the number of alcohol reinforcers earned, despite differences in rate of responding. $\boldsymbol{a}$, All genotypes received equal access to alcohol reinforcers across acquisition. $\boldsymbol{b}$, Access to alcohol relative to body weight was comparable across groups. The average grams per kilogram consumed per session was equivalent across groups (inset). $c, X Y$ mice and mice who developed testes made more active nosepokes per minute than did XX mice and mice that developed ovaries. *Interaction effect $X Y$ chromosome and male gonad, $p<0.05$. Error bars represent SEM. Post hoc analyses conducted with Tukey's HSD. XYM $n=16, X Y F n=12$, $\mathrm{XXM} n=14, \operatorname{XXF} n=13$.

tion on the number of inactive nosepokes ( $p$ values $>0.1$ ). Mice clearly discriminated between the active and inactive nosepokes (data not shown).

\section{Conditioned taste aversion}

Devaluation was assessed by measuring alcohol consumption during lithium chloride-induced devaluation and after the extinction test (see below). A mixed-model ANOVA revealed an interaction between devaluation status and day of CTA, indicating that only the devalued mice decreased alcohol consumption across the $3 \mathrm{~d}$ of LiCl-induced CTA training $\left(F_{(2,147)}=\right.$ $22.47, p<0.001)$. a

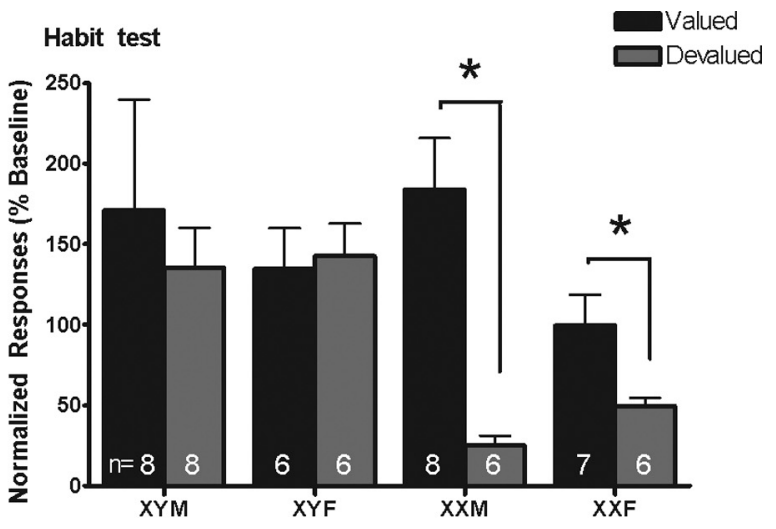

b

Post-test consumption



\begin{tabular}{|c|c|c|c|c|}
\hline Chromosome & $\sigma^{7}$ & $\sigma^{2}$ & 우 & P \\
\hline Gonad & $\sigma^{2}$ & 오 & $\sigma^{2}$ & P \\
\hline
\end{tabular}

Figure 2. XY mice showed more rapid habit formation to alcohol, regardless of gonadal phenotype. $\boldsymbol{a}$, After moderate training, XY mice were insensitive to alcohol devaluation, indicating habitual responding. XX mice remained goal-directed. ${ }^{* X X M}$ and XXF devalued mice make fewer active nosepokes than valued mice after devaluation. $p<0.05$. $\boldsymbol{b}$, Devalued mice consumed less of the alcohol reinforcer in a 30 min consumption test. Gonadal females consumed more alcohol than gonadal males in this ad libitum context. $p<0.05$. Error bars represent SEM. Post hoc analyses conducted with Tukey's HSD. Valued: XYM $n=8, \mathrm{XYF} n=6, \mathrm{XXM}$ $n=8, \mathrm{XXF} n=7$. Devalued XYM $n=8, \mathrm{XYF} n=6, \mathrm{XXM} n=6, \mathrm{XXF} n=6$.

Habit test and posttest consumption

A four-way ANOVA on normalized active nosepoke responding during the habit test revealed a main effect of devaluation and sex chromosome complement $\left(F_{(1,32)}=4.31, p=0.04, F_{(1,32)}=4.22\right.$, $p=0.04)$. Both valued and devalued $\mathrm{XY}$ mice responded to the same degree during the habit test, while devalued XX mice responded less than valued XX mice, indicating that chromosomal male mice were behaving in a habitual manner, while chromosomal female mice were goal-directed (Fig. $2 a$ ). There was no effect of gonadal sex or GDX on habit formation ( $p$ values $>0.3$ ), so the data were collapsed across these factors for presentation.

To verify the effectiveness of the CTA training on the day of habit testing, mice were given $30 \mathrm{~min}$ access to $10 \%$ alcohol in an empty cage following the habit test. A four-way ANOVA analyzing the amount of alcohol consumed per $\mathrm{kg}$ of body weight revealed a main effect of devaluation and a main effect of gonadal sex $\left(F_{(1,37)}=59.09, p<0.001, F_{(1,37)}=4.38, p=0.043\right)$, indicating that the CTA procedure successfully devalued alcohol by specifically reducing consumption in the devalued group. Additionally, gonadal females consumed more alcohol than gonadal males during the 30 min test (Fig. $2 b$ ) with no effect of sex chromosome complement $(p>0.7)$. There was a nonsignificant interaction effect of gonadectomy $\left(F_{(1,37)}=3.41, p=0.073\right)$. 


\section{Locomotor behavior}

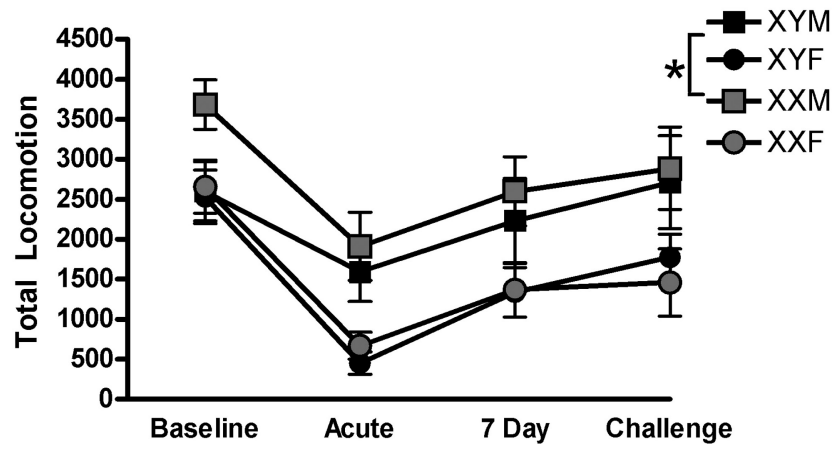

Figure 3. At baseline, locomotor activity is highest in XXM mice. After acute, chronic, or challenge alcohol injections after withdrawal, locomotor behavior is mediated by gonadal sex. ${ }^{*}$ Gonadal males are more active after alcohol exposure. $p<0.05$. Error bars represent SEM. Post hoc analyses conducted with Tukey's HSD. XYM $n=9, \operatorname{XYF} n=9, \operatorname{XXM} n=10, \operatorname{XXF} n=10$.

\section{Locomotor behavior}

A mixed-model ANOVA revealed an interaction effect of gonadal sex and chromosome complement on locomotor behavior $\left(F_{(1,219)}=4.08, p<0.045\right)$. There was nonsignificant interaction effect of gonadectomy, gonadal sex and testing condition $\left(F_{(1,219)}=2.91, p=0.089\right)$. Post hoc analyses indicated that XXM mice were more active during baseline assessment than other genotypes. However, when alcohol was on board, gonadal male mice had higher locomotor activity. In addition, ANOVA revealed a main effect of testing condition on locomotor behavior $\left(F_{(3,219)}=22.17, p<0.001\right)$. Post hoc analyses indicated that locomotor activity was significantly lower than baseline after acute, chronic and challenge alcohol exposure $(p<0.05)$. Additionally, locomotor activity after chronic exposure was greater than after acute exposure ( $p<0.05$; Fig. 3 ). Locomotor behavior after chronic exposure did not differ from activity after challenge exposure $(p>0.05)$.

\section{Discussion}

We found that sex chromosome complement and not gonadal phenotype determined alcohol habit formation (Fig. 2a): XY mice (XYM and XYF) were not sensitive to reinforcer devaluation and responded at levels comparable to those before devaluation, indicating habitual behavior. In contrast, XX mice (XXM and XXF) were sensitive to devaluation, demonstrating goaldirected behavior. Neither gonadal phenotype nor gonadectomy predicted whether mice responded in a habitual or goal-directed manner during the habit test, confirming that sex chromosome complement rather than gonadal status influenced behavior. Conversely, in an ad libitum context, gonadal females consumed more alcohol than gonadal males (Fig. $2 b$ ) with no effect sex chromosome complement. A trend toward a gonadectomy by gonadal sex interaction suggests an organizing and activating influence of hormones on free alcohol drinking. These data are consistent with several reports indicating greater free alcohol consumption in female rodents (Lancaster et al., 1996; Middaugh et al., 1999). The consumption test also confirmed that the alcohol reinforcer was successfully devalued only in mice that underwent alcohol CTA. These data suggest that the habitual responding observed in the XY (XYM/XYF) mice could not be attributed to deficits in learning/memory of alcohol devaluation.

Research has demonstrated that reinforcer exposure, rather than the number of responses made, predicts habit formation (Adams, 1982). Importantly, there were no differences in the number of alcohol reinforcers earned during acquisition (Fig. $1 a)$, though chromosomal males (XYF/XYM) had higher response rates than chromosomal females (XXF/XXM) and mice with testes (XYM/XXM) had higher response rates than those without $(\mathrm{XXF} / \mathrm{XYF})$, as shown in Figure 1c. This difference in response efficiency, whereby some groups make more responses but receive equal access to reinforcer, may reflect differences in motivation to work for alcohol reinforcer or disparity in the ability to track reinforcer availability. Additionally, the chromosome-mediated differences in habitual responding did not appear to be explained by differences in locomotor behavior. Locomotor activity at baseline was determined by an interaction between gonadal and chromosomal sex such that XXM mice made more beam breaks than other genotypes. While we did not observe an increase in activity in response to ethanol in this strain of mouse (MF1), after both acute and chronic exposure to alcohol, mice that developed testes (XYM/XXM) made more beam breaks during the session than those without. A nonsignificant trend toward an interaction between testing condition, gonadal sex and gonadectomy, indicates that the locomotor effects of alcohol are not mediated by sex chromosome complement, but rather a potential interaction between organizational and activational effects of gonadal hormone in which testicular hormones predict increased locomotor activity (Fig. 3).

Together, these data suggest that sex chromosome complement influences habit formation for alcohol, independently of gonadal phenotype and hormone circulation, in such a way that male chromosomal status predicts rapid alcohol habit formation. These findings stand in contrast to our data with "natural" reinforcers (Quinn et al., 2007) where habit formation for sucrose was more rapid in XX than XY mice. Though our data do not distinguish between the pharmacological effects and caloric value of alcohol, the direction of the sex differences in the rate of habit formation of alcohol stands in direct contrast to that of habit for a sucrose reinforcer similar in caloric value, indicating that caloric value alone cannot explain the observed effect of sex chromosome complement on behavior. Sex chromosome complement, therefore, differentially affects habit formation depending on the reinforcer, possibly explaining differences in rates of dependence on various drugs and foods in men and women.

It has been reported that alcohol habits form more rapidly than habits for natural reinforcers in male animals (Dickinson et al., 2002), but the rate of alcohol habit formation in female rodents has not been previously studied. The shift in response strategy from goal-directed action to stimulus-response habit is thought to result from a transition from ventral striatal circuitry including prefrontal cortex to a more dorsal circuit involving dorsolateral striatum (Everitt and Robbins, 2005), and alcohol may interact with this corticostriatal circuitry to influence the rate of progression from goal-directed to habitual responding. For example, alcohol exposure can produce impaired prefrontal cortical function (Sullivan and Pfefferbaum, 2005) and possibly disrupt input to the ventral striatum (Szumlinski et al., 2007). Additionally, alcohol exposure has been shown to increase activation of the NR2B subunit of NMDA receptors in the dorsolateral striatum, producing enhanced signaling consistent with habit formation (Wang et al., 2007). Furthermore, bath application of alcohol on striatal slices results in decreased long-term potentiation in the dorsomedial striatum, where decreased activity is associated with habit formation (Yin et al., 2007). Work from Devaud et al. (2000) also found that gonadectomy did not influence sex-specific effects of alcohol on NMDA or $\mathrm{GABA}_{\mathrm{A}}$ receptors in cortex indicating that activational influences of hor- 
mones were not a factor; however, their study cannot distinguish whether genetic or organizational hormonal factors were involved. Our data suggest that sex chromosomes, independent of gonadal hormones, mediate sex differences in habit formation for alcohol and may underlie sex differences in alcohol-induced neuroadaptations.

How genetic factors influence the shift from goal-directed behaviors to habit is only beginning to be understood. A recent study from Yu et al. (2009) demonstrated that the absence of $A_{2 A}$ adenosine receptors in the striatum impaired the development of habitual responding for food. Additionally, work from Hilário et al. (2007) has indicated a role for endocannabinoid signaling in habit development through the use of a cannabinoid receptor type 1 (CB1) knock-out mouse. Both $\mathrm{A}_{2 \mathrm{~A}}$ and $\mathrm{CB} 1$ receptors are thought to interact with dopamine receptors as receptor heteromers to modulate neurotransmission in the striatum (Ferré et al., 2009) and may influence dopamine receptor-mediated behaviors (Martín et al., 2008; Hsu et al., 2010). Notably, no studies have examined genetic factors involved in alcohol habits, and how alcohol may act at these synapses to influence long-term plasticity.

It is becoming evident that sex chromosomes play a key role in the regulation of plasticity-associated signaling cascades (Jazin and Cahill, 2010), which may underlie shifts in corticostriatal function. Sex differences in striatal circuitry have been shown to result from a combination of gonadal and chromosomal influences. Sex chromosome complement has been shown to influence $\mathrm{TH}$ expression in dopaminergic neurons in vitro (Carruth et al., 2002). It has also been shown that sex chromosome complement can cause differential gene expression in the adult striatum-in vivo (Chen et al., 2009). Collectively, these studies suggest that sex chromosome-mediated differences in genes that regulate synaptic plasticity within the striatum may underlie functional sex differences in the development of habits. Study of $\mathrm{Y}$ chromosome genes, genes that escape $\mathrm{X}$ chromosome inactivation and their paralogues on the $\mathrm{Y}$ chromosome may provide a starting point for exploration of genetic sex differences. Further investigation into the precise neurobiological factors that mediate the divergence in habit formation caused by male and female chromosomal status, and the influence of alcohol consumption, is critical for developing gender-specific treatment and early intervention methods for individuals suffering from and at-risk for developing alcohol dependence.

\section{References}

Adams CD (1982) Variations in the sensitivity of instrumental responding to reinforcer devaluation. Q J Exp Psychol B 34:77-98.

Arnold AP, Chen X (2009) What does the "four core genotypes" mouse model tell us about sex differences in the brain and other tissues? Front Neuroendocrinol 30:1-9.

Arnold AP, Gorski RA (1984) Gonadal steroid induction of structural sex differences in the central nervous system. Annu Rev Neurosci 7:413-442.

Belin D, Everitt BJ (2008) Cocaine seeking habits depend upon dopaminedependent serial connectivity linking the ventral with the dorsal striatum. Neuron 57:432-441.

Carruth LL, Reisert I, Arnold AP (2002) Sex chromosome genes directly affect brain sexual differentiation. Nat Neurosci 5:933-934.

Chen X, Grisham W, Arnold AP (2009) X chromosome number causes sex differences in gene expression in adult mouse striatum. Eur J Neurosci 29:768-776.
Devaud LL, Morrow AL, Nguyen UT (2000) Ovariectomy has minimal effects on neuroadaptations associated with ethanol dependence in female rats. Neurochem Int 37:433-442.

De Vries GJ, Rissman EF, Simerly RB, Yang LY, Scordalakes EM, Auger CJ, Swain A, Lovell-Badge R, Burgoyne PS, Arnold AP (2002) A model system for study of sex chromosome effects on sexually dimorphic neural and behavioral traits. J Neurosci 22:9005-9014.

Dickinson A (1985) Actions and habits: the development of behavioural autonomy. Philos Trans R Soc Lond B 308:67-78.

Dickinson A, Wood N, Smith JW (2002) Alcohol seeking by rats: action or habit? Q J Exp Psychol B 55:331-348.

Everitt BJ, Robbins TW (2005) Neural systems of reinforcement for drug addition: from actions to habits to compulsion. Nat Neurosci 8:1481-1489.

Ferré S, Goldberg SR, Lluis C, Franco R (2009) Looking for the role of cannabinoid receptor heteromers in striatal function. Neuropharmacology $56: 226-234$.

Hilário MR, Clouse E, Yin HH, Costa RM (2007) Endocannabinoid signaling is critical for habit formation. Front Integr Neurosci 1:6.

Hsu CW, Wang CS, Chiu TH (2010) Caffeine and a selective adenosine A2A receptor antagonist induce sensitization and cross-sensitization behavior associated with increased striatal dopamine in mice. J Biomed Sci 17:4.

Jazin E, Cahill L (2010) Sex differences in molecular neuroscience: from fruit flies to humans. Nat Rev Neurosci 11:9-17.

Jentsch JD, Taylor JR (1999) Impulsivity resulting from frontostriatal dysfunction in drug abuse: implications for the control of behavior by reward-related stimuli. Psychopharmacology (Berl) 146:373-390.

Kalaydjian A, Swendsen J, Chiu WT, Dierker L, Degenhardt L, Glantz M, Merikangas KR, Sampson N, Kessler R (2009) Sociodemographic predictors of transitions across stages of alcohol use, disorders, and remission in the National Comorbidity Survey Replication. Compr Psychiatry 50:299-306.

Lancaster FE, Brown TD, Coker KL, Elliott JA, Wren SB (1996) Sex differences in alcohol preference and drinking patterns emerge during the early postpubertal period. Alcohol Clin Exp Res 20:1043-1049.

Lynch WJ, Roth ME, Carroll ME (2002) Biological basis of sex differences in drug abuse: preclinical and clinical studies. Psychopharmacology (Berl) 164:121-137.

Martín AB, Fernandez-Espejo E, Ferrer B, Gorriti MA, Bilbao A, Navarro M, Rodriguez de Fonseca F, Moratalla R (2008) Expression and function of CB1 receptor in the rat striatum: localization and effects on D1 and D2 dopamine receptor-mediated behaviors. Neuropsychopharmacology 33:1667-1679.

Middaugh LD, Kelley BM, Bandy AL, McGroarty KK (1999) Ethanol consumption by C57BL/6 mice: influence of gender and procedural variables. Alcohol 17:175-183.

Quinn JJ, Hitchcott PK, Umeda EA, Arnold AP, Taylor JR (2007) Sex chromosome complement regulates habit formation. Nat Neurosci 10:1398-1400.

Sullivan EV, Pfefferbaum A (2005) Neurocircuitry in alcoholism: a substrate of disruption and repair. Psychopharmacology (Berl) 180:583-594.

Szumlinski KK, Diab ME, Friedman R, Henze LM, Lominac KD, Bowers MS (2007) Accumbens neurochemical adaptations produced by binge-like alcohol consumption. Psychopharmacology 190:415-431.

Wang J, Carnicella S, Phamluong K, Jeanblanc J, Ronesi JA, Chaudhri N, Janak PH, Lovinger DM, Ron D (2007) Ethanol induces long-term facilitation of NR2B-NMDA receptor activity in the dorsal striatum: implications for alcohol drinking behavior. J Neurosci 27:3593-3602.

Yin HH, Park BS, Adermark L, Lovinger DM (2007) Ethanol reverses the direction of long-term synaptic plasticity in the dorsomedial striatum. Eur J Neurosci 25:3226-3232.

Yu C, Gupta J, Chen JF, Yin HH (2009) Genetic deletion of $A_{2 A}$ adenosine receptors in the striatum selectively impairs habit formation. J Neurosci 29:15100-15103.

Zou H, Xie Q, Zhang M, Zhang C, Zhao G, Jin M, Yu L (2009) Chronic alcohol consumption from adolescence-to-adulthood in mice- effect on growth and social behavior. Drug Alcohol Depend 104:119-125. 